\title{
Self-organization of topological defects for a triangular-lattice magnetic dots array subject to a perpendicular magnetic field
}

\author{
R.S. Khymyrn, V.E. Kireev², B.O. Ivanov ${ }^{2}$ \\ 1 Oakland University, 48309 Rochester Hills, MI, USA \\ 2 Institute of Magnetism, 03142 Kiev, Ukraine
}

Received April 18, 2014, in final form May 15, 2014

\begin{abstract}
The regular array of magnetic particles (magnetic dots) of the form of a two-dimensional triangular lattice in the presence of external magnetic field demonstrates complicated magnetic structures. The magnetic symmetry of the ground state for such a system is lower than that for the underlying lattice. Long range dipole-dipole interaction leads to a specific antiferromagnetic order in small fields, whereas a set of linear topological defects appears with the growth of the magnetic field. Self-organization of such defects determines the magnetization process for a system within a wide range of external magnetic fields.
\end{abstract}

Key words: magnetic dot, topological defect

PACS: $75.10 . H k, 75.50 . T t, 75.30 . K z$

\section{Introduction and motivation}

Self-organization phenomena are usually associated with complex systems far from equilibrium 113]. In that case, the standard approach, depicting condensed matter as a gas of weakly interacting quasiparticles (phonons, magnons, etc.) obtained within the framework of linearized theory, may become inappropriate, and the main role is played by soliton-type excitations [3, 4]. One can point out numerous examples of such a behavior in very different physical systems. In particular, the Berezinskii-KosterlitzThouless phase transition in quasi-two-dimensional magnets is driven by the appearance of a finite density of free magnetic vortices [5-7]. Plastic deformation of solids may lead to the emergent complexity of a defect structure, resulting in a hierarchy of super-defects such as dislocation and disclination systems [8]. A fast quench across the phase transition line may produce a finite density of frozen topological defects of various types; such a behavior is known for a wide range of systems from all fields of physics, either for classical [9] or for quantum [10] systems. Note the defect line scenario of phase transitions for two-dimensional systems with discrete symmetry breaking, see, for example, [11]. The common feature of all the above examples is a considerable role of stochastic factors, connected primarily to thermal fluctuations, which leads to an irregular distribution of defects [9, 12].

Magnetic ordering is usually attributed to the exchange interaction of atomic spins, leading to rather simple magnetically ordered states [13]. Long-range magnetic dipole interaction usually produces smooth non-uniformity (domain structures of different kinds) above this simple exchange structure [14-16]. However, the theoretical investigation of the systems of magnetic moments with pure dipolar interaction, the so-called dipolar magnets, shows that many physical properties, lacking in the spin-exchanged systems, are present for those systems. We should first note the presence of a non-unique ground state with nontrivial continuous degeneracy for quite simple bipartite lattices, such as three-dimensional cubic lattice, [17, 18] and for a two-dimensional square lattice, [19-21] as well as specific phase transitions induced by an external magnetic field [22-24]. 
The models of dipolar magnets were originally discussed in regard to real crystalline spin systems. A renewed interest to such models has been caused by investigation of two-dimensional lattices of submicron magnetic particles (the so-called magnetic dots), see [25-27] for a recent review. The ability to create the dots with practically exactly equal sizes and precisely controlled distance between them, leads to the long-range order phenomena and can be treated as the creation of artificial crystals.

A direct exchange interaction between the dots is negligible, and the dipolar interaction is the sole source of coupling between the dots. For small enough dots of a size smaller than $100 \mathrm{~nm}$, the magnetization inside a dot is almost uniform, producing the total magnetic moment $m_{0} \gg \mu_{\mathrm{B}}$, where $\mu_{\mathrm{B}}$ is the Bohr magneton, i.e., the typical value for an atomic magnetic moment. For rather small magnetic dots of volume $10^{3} \div 10^{5} \mathrm{~nm}^{3}$, the value of $m_{0}$ exceeds $10^{4} \mu_{\mathrm{B}}$, and for dense arrays the characteristic energy is higher than the energy of thermal motion at room temperature [22, 23, 28]. Moreover, the MerminWagner theorem is not valid for two-dimensional magnets with a dipolar coupling of spins having continuous degeneracy, and a true long range order can exist even for a purely two-dimensional case at finite temperatures, either for ferromagnets [29, 30] and antiferromagnets [31]. Thus, one can expect that thermal effects are less important for magnetic dot arrays up to high temperatures compared with the Curie temperature of the magnetic material.

Such systems represent dipolar magnets and fill their theoretical investigation with a new physical content. Owing to the absence of exchange, magnetic dot arrays constitute a promising material for high-density magnetic storage media. For this purpose, the dense arrays of small enough magnetic dots with magnetic moments perpendicular to the array plane are optimal, see Refs. [32, 33, 35]. Currently, the ordered arrays of magnetic sub-micron elements have been discussed as materials for the so-called magnonics. In this new field in the applied physics of magnetism, magnon modes with a discrete spectrum present for magnetic nanoelements are used for processing the microwave signals [37]. Novel prospects are opened by the observation of the excitation of collective spin oscillations by femtosecond laser pulses [34,36]. Magnon spectra for dot arrays demonstrate a non-analytic behavior either for small wavevectors, [38-41] or at some symmetrical points within the Brillouin zone [40]. Non-reciprocal effects for magnetic dot lattices [42, 43] and non-trivial properties of the magnon modes localized on the defects of dot arrays have been recently found [44]. The presence of phase transitions opens novel opportunities for design of magnonic devices with the band structure operated by external parameters, e.g., magnetic field, see for the recent review [45]. Thus, magnetic dot arrays are interesting as radically new objects for both the fundamental and applied physics of magnetism.

If the magnetic moment of an individual magnetic particle is perpendicular to the array plane ( $x y$ plane), $\mathbf{m}= \pm m_{0} \mathbf{e}_{z}$, the system can be described on the basis of the Ising model. The energy of dipolar interaction of Ising moments perpendicular to the system's plane is minimal for antiparallel orientation of magnetic moments. Within the nearest-neighbors approximation, such interactions lead to antiferromagnetic (AFM) structures, e.g., simple chessboard AFM ordering is known for a two-dimensional Ising square lattice with dipolar interaction [22, 27]. However, the close-packed triangular lattices of the magnetic dots are also frequently used in experiments. In particular, these lattices of cylindrical particles considerably extended in the direction normal to the array plane are naturally obtained when the array is prepared by controlled self-organization [46]. However, the triangular lattice is not bipartite. Such a lattice with AFM interaction of the moments is a typical example of frustrated antiferromagnets [47]. It is worth mentioning here that the behavior of a dot array with in-plane anisotropy is essentially different from our case. In this case, the dipolar interaction is not clearly AFM; and for a model of infinite unbounded array, the ferromagnetic state is stable [21], whereas for finite array, there appears a mesoscopic non-uniform state of a form of either domain wall [48] or magnetic vortex [48, 49].

For a nearest-neighbor Ising triangular lattice with AFM interaction, thermodynamic properties are quite unusual [47]. It is enough to mention that in this model there is no magnetic ordering at any finite temperature $T \neq 0$; the ordering appears as a result of accounting for the next-nearest-neighbor interactions only [50-52]. This counterintuitive feature can be explained within the concept of creation of linear topological defects with zero energy [53]. For the case of magnetic dipole interaction, (common to what is observed for many next-nearest-neighbor interaction models), our analysis shows the presence of AFM ordering for small enough magnetic fields.

In the present work, a cascade of phases with different patterns of dot magnetization has been found for a triangular lattice of mesoscopic magnetic dots with perpendicular magnetization and in an external 
magnetic field also perpendicular to the plane of the dot lattice. The transition between these states is governed by a novel mechanism involving the creation of an ordered system of linear topological defects with non-zero magnetization. For those transitions, thermal fluctuations are insignificant and one can expect quite regular spatial distribution of such defects.

\section{Model description}

Consider a system of magnetic moments of magnetic particles $\mathbf{m}_{\mathbf{n}}$ placed in the sites of a triangular lattice $\mathbf{n}$, parallel to the $x y$-plane,

$$
\mathbf{n}=a k \mathbf{e}_{x}+\frac{a l}{2}\left(\mathbf{e}_{x}+\sqrt{3} \mathbf{e}_{y}\right)
$$

where $a$ is a lattice constant, $k, l$ are integers, and $\mathbf{e}_{x}$ and $\mathbf{e}_{y}$ are unit vectors parallel to $x$ and $y$ axes, respectively. We assume that any particle has easy-axial (Ising-like) anisotropy of the form of $w_{\mathbf{n}, a}=$ $H_{a}\left(m^{2} \mathbf{n}, x+m^{2} \mathbf{n}, y\right) / 2 m_{0}$, and that the corresponding internal anisotropy field $H_{a}$ is substantially higher than the characteristic field of the dot dipolar interaction, $H_{*}=m_{0} / a^{3}$. Such systems of magnetic particles made of soft ferromagnets, with the $H_{a}$ originating from the shape anisotropy $H_{a} \sim 2 \pi M_{\mathrm{s}}$, where $M_{\mathrm{s}}$ is the saturation magnetization of the material, are used for magnetic memory applications [35]. The interaction field $H_{*}$ is proportional to a small geometric factor, $H_{*}=M_{\mathrm{s}}\left(v_{0} / a^{3}\right)$, where $\nu_{0}$ is the dot volume. This interaction field can be much less than $H_{a}$ even for dense enough lattices [22]; and it is not capable of deflecting the dot magnetic moment from the $z$-axis. Thus, we can assume that all magnetic moments are $\mathbf{m}_{\mathbf{n}}=m_{0} \mathbf{e}_{z} \sigma_{\mathbf{n}}$, where $\sigma_{\mathbf{n}}= \pm 1$. Then, the Hamiltonian of this system of magnetic moments can be written as

$$
W=m_{0}^{2} \sum_{\mathbf{n} \neq \mathbf{n}^{\prime}} \frac{\sigma_{\mathbf{n}} \sigma_{\mathbf{n}^{\prime}}}{\left|\mathbf{n}-\mathbf{n}^{\prime}\right|^{3}}-m_{0} H \sum_{\mathbf{n}} \sigma_{\mathbf{n}},
$$

where an external magnetic field $\mathbf{H}=H \mathbf{e}_{z}$ is applied perpendicularly to the plane of the array. The first term describes dipolar interaction, with the summation performed over all of the pairs of the lattice sites. Below, for the sake of simplicity, we present the energy (per one magnetic particle) in the units of $m_{0}^{2} / a^{3}$ and we use the dimensionless magnetic field, $h=H / H_{*}$, where the characteristic value $H_{*}=m_{0} / a^{3}$. The present model applies directly to any triangular lattice of identical dipoles that are restricted to the two directions of normal orientation [21]. It is interesting that the model formulated in this paper can be used to describe a system of vortex state magnetic dots [22] accounting for the interaction of a magnetic moment of vortex cores. The direction of the core moments is connected with the topological invariant, and the vortices with upward or downward directions of the moments survive until non-small values of magnetic field [54] are reached.

As has been mentioned above, two sources of degeneracy are present in our system, and it is not $a$ priori obvious which structure will constitute the ground state. In this situation it is natural to start with the numerical analysis of the problem.

\section{Ground states for infinite system: Numerical analysis}

To find the global minimum of the energy of a system, there was used a Monte-Carlo (MC) approach combined with a simulated annealing (SA) method, see the textbook [55] for details. The standard MC method is based on the attempts to reverse the moment on the random site, and the only reversals favorable to energy are allowed. By contrast, for MC-SA, the probability of the reversal is non-zero even if the energy grows after the reversal; otherwise, the system with a high probability will be "frozen" in some local minimum. The probability depends not only on the energy gain $\Delta E$ but also on the global parameter $T$ called the temperature. If the reversal is favorable in the energy, the moment is always reversed, irrespective of $T$. But even if the reversal is unfavorable, the non-zero probability of a reversal is chosen as follows: flip-over takes place if $\Delta E<T|\log p|$, where $T$ is the current value of temperature, $p$ is a random value $0<p \leqslant 1$. Here, the parameter temperature determines the strategy of minimization and the meaning of the temperature is the same as for annealing in metallurgy involving initial heating and controlled cooling of a material, thereby avoiding the formation of defects. $T$ changes according to the quantity of 
full steps of MC-SA such that the initial temperature is high enough compared with the interaction energy, and then the temperature decreases. For a concrete analysis, we took the rhombus-shaped samples with consecutively increasing periods up to $16 \times 16$ and used periodic boundary conditions.

\subsection{Simplest ground states of the system: zero field and saturation}

For a triangular Ising lattice in a several-neighbor approximation, a simple AFM order with two sublattices can be implemented [56]. We found the same configuration for a long-range dipolar interaction in the absence of the field and for a small enough magnetic field. For these states, the magnetic elementary cell is rectangular having lower symmetry than for the underlying triangular lattice, and this state possesses a much higher discrete degeneracy than a simple chessboard structure for a square lattice discussed before. Several AFM states can occur in a system, which are different but fully equivalent by their energies. Figure 1 presents three of these states, while the other three states are obtained from them by changing the magnetic moment sign $\sigma_{\mathbf{n}}$ at all of the particles. Note the deviation from the standard AFM case with the antiparallel orientation of all the nearest neighbors, which is a typical manifestation of frustration in a system.
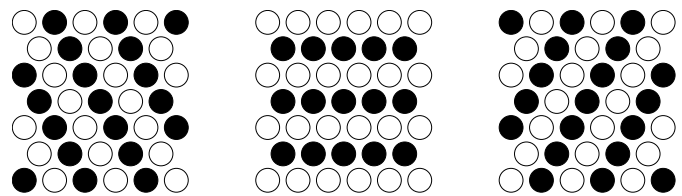

Figure 1. Uniform antiferromagnetic states giving the energy minimum at a small magnetic field. Here and below, in all figures, the open and closed circles denote the particles with the upward and downward moments, respectively.

\subsection{Monte-Carlo analysis for intermediate field values}

MC-SA analysis shows that for some small but finite values of the magnetic field, at least up to $h=0.7$, the mean value of the magnetic moment $\langle m\rangle$ equals zero, which indicates a simple AFM structure. For higher fields, numerous more complex structures with $0<\langle m\rangle\left\langle m_{0}\right.$ occur in the intermediate region between AFM state and saturated state. The mean value of the magnetic moment (per one particle) $\langle m\rangle$ corresponding to these configurations, is present in figure 2.

Note the specific regions of this dependence present at different field intervals; first, the region with small values of $\langle m\rangle \leqslant 0.2 m_{0}$ having a rather non-regular dependence of $\langle m\rangle$ on $h$; second, the regions with constant values of $\langle m\rangle$ independent of the magnetic field (shelves); and third, the saturation region. The characteristic magnetic structures found in these regions are depicted in figure 3 .

Monte-Carlo data are not too clear in the region of small fields such as $0.7 \div 0.9$, and the magnetic structures are far from the simple AFM structures, see figures $3(\mathrm{a}), 3(\mathrm{~d})$. Within this region, the aforementioned structure with a set of topological defects appears. A detailed discussion of this region is the main issue of our article.

Within the shelf regions, almost all initial Monte-Carlo configurations lead to the same magnetic structures, which correspond to the formation of triangular superlattices for the minority dots (antiparallel to the magnetic field) with different lattice spacings. As an example, note the ideal triangular superlattices with $\langle m\rangle=m_{0} / 3$ and with the period $a_{\mathrm{sl}} / a=\sqrt{3}$, see figure $3(\mathrm{f})$ present at the values $2.4 \lesssim h \lesssim 6.4$. For higher fields, the superlattices with $a_{\mathrm{sl}} / a=2$ [figure 3(h)], $a_{\mathrm{sl}} / a=\sqrt{7}$, [figure 3(i)] and $a_{\mathrm{sl}} / a=3$ [in figure 3(j)] correspond to such shelves. For high magnetic fields near the saturation region, the magnetization process is going through creating a superlattice of flipped dots of small density.

In the region of low magnetic fields, as well as in the regions of a magnetic field where the transitions between the superlattices occur, resettability of Monte-Carlo result lowers, and the results become unreliable. The observed magnetic structures in these transition regions are characterized by much lower symmetry than for the shelf regions. For example, at the values $0.9 \lesssim h \lesssim 1.5$, where the finite (but small) 


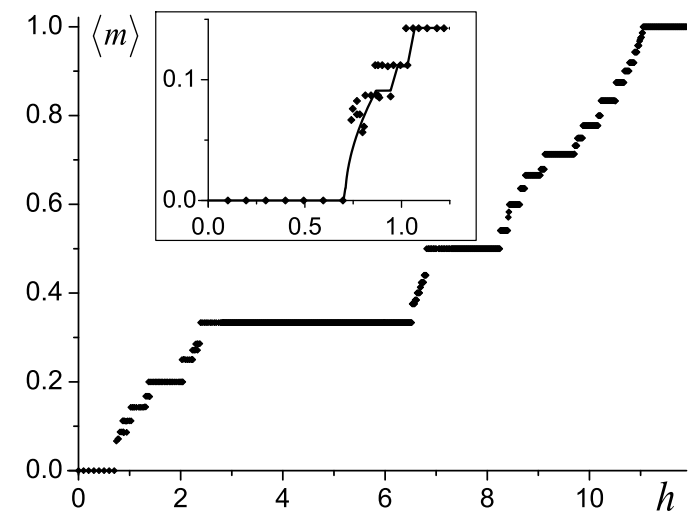

Figure 2. The mean value of magnetization $\left\langle m_{0}\right\rangle$ (in units of $m_{0}$, per one dot) of the array as a function of magnetic field (in units of $H_{*}=M_{0} / a^{3}$ ) found by Monte-Carlo simulations. Magnetization function at low fields found by an exhaustive search of the states of rhombus-shaped samples (full line) together with the Monte-Carlo data (symbols) are presented in the insert.

magnetic moment $\left\langle m_{0}\right\rangle$ is formed, the translational symmetry for a set of flipped dots cannot be attributed to a simple superlattice structure, see figure 3(a) However, in this figure one can clearly see a novel element, an additional zigzag line of the sites oriented parallel to one of the translation vectors of the lattice. The resulting magnetic structure can be interpreted as an antiferromagnetic domain structure in a system with a zigzag line as a domain wall. Such topological linear defects were described for a two-sublattice antiferromagnetic state with an interaction of a few neighboring moments [57]. For the region of small fields, an increase of magnetic field leads to an increase of the density of topological linear defects, see figures $3(\mathrm{a}), 3(\mathrm{c})$. A minimal field for the start of this process corresponds to a low density of such defects, and to find the critical field one needs to consider larger and larger systems. Namely, to present a stripe of width $n$ we need a system of at least $(2 n+1) \times(2 n+1)$ size. Below, in section 4 we find the starting field for the creation of a set of topological defects by an analytical calculation.

To refine the Monte-Carlo data and to clarify the magnetic states at the fields of interest, $0 \leqslant h \leqslant 3$, we

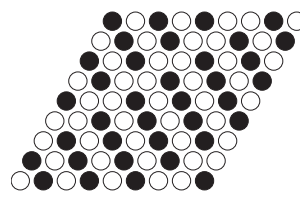

(a) $h=1.0$

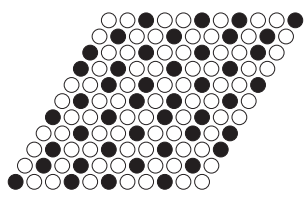

(e) $h=2.3$

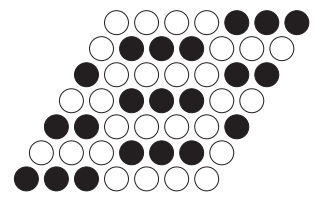

(b) $h=1.1$

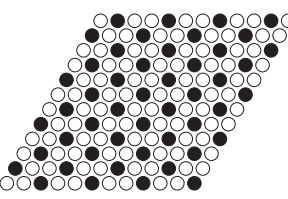

(f) $h=4.0$

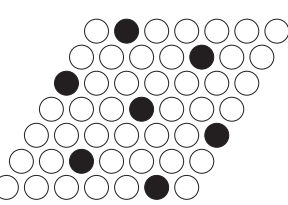

(i) $h=9.5$

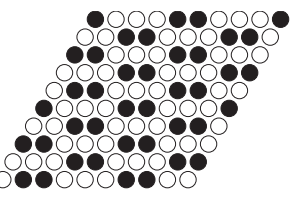

(c) $h=1.5$

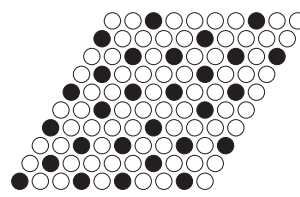

(g) $h=6.77$

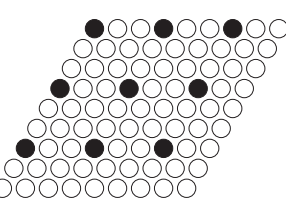

(j) $h=10.1$

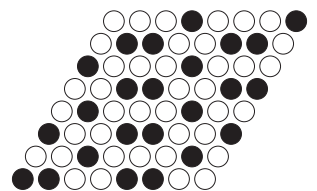

(d) $h=2.1$

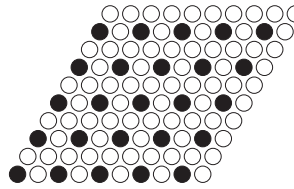

(h) $h=7.5$

Figure 3. Ground states for characteristic values of magnetic field found by Monte-Carlo simulations. 
perform a direct exhaustive search based on the picture of stripe AFM domain structures for rhombusshaped space regions with various (not necessarily equidistant) geometries of domain lines, up to the size $60 \times 60$. It appears that for all fields the only equidistant structure corresponds to the minimal configurations, with linear system of stripes at $h<1.5$ or triangular superlattice at $h>2.0$. Then, the only equidistant structures with the size up to $300 \times 300$ were examined. The magnetization curve based on these calculations is represented above in the insert in figure 2 and is compared with the Monte-Carlo data.

The common "topological" scenarios are present for other transition regions, both below and above the shelf regions with the magnetic structure of a form of ideal triangular superlattices of minority dots. For example, the structure present at $2.0 \lesssim h \lesssim 2.4$ can be described as a "compression" of the domains of the superlattice of period $a \sqrt{3}$ by the lines of dots with down magnetic moments, see figures $3(\mathrm{~d})$ and $3(\mathrm{e})$, whereas the state at the opposite end of this shelf can be seen as a "rarefication" of the $a \sqrt{3}$ superlattice, see figure $3(\mathrm{~g})$. The transition structures corresponding to the "higher" shelves have $C_{6}$ symmetry, higher than for low field structures.

\section{Magnetic ground states: Analytic description}

For a non-frustrated square lattice of Ising magnetic moments, the destruction of both types of states is started through the creation of a point defect in the state, the single magnetic dot with the magnetic moment being reversed with respect to the regular structure of a given state [22]. First, in this section we check the validity of such a scenario for saturated and AFM states of a triangular lattice array of magnetic dots. Then, there follows a theoretical description of the novel topological mechanisms.

\subsection{Point defect scenarios}

In order to determine the values of the magnetic fields which correspond to "point defect instability" we have calculated the change in dipolar interaction energy that occurs when the magnetic moment of a single dot is reversed with respect to the ferromagnetic and two-sublattice AFM structures [22]. This energy change is determined by the energy per dot in the initial states, which can be expressed by simple lattice sums calculated with high precision. These sums here and below were calculated using a standard software package Mathematica.

The point defect scenario well describes the instability of the saturated state. It is easy to see that the change of the energy of the saturated state with the flip of a single magnetic moment can be presented as $W_{1}=2 m_{0}\left(H-H_{\text {sat }}\right)$, where

$$
H_{\text {sat }}=m_{0} \sum_{\mathbf{n}_{i} \neq 0} \frac{1}{\left|\mathbf{n}_{i}\right|^{3}} \equiv h_{\text {sat }} \frac{m_{0}}{a^{3}}, \quad h_{\text {sat }} \approx 11.034176 .
$$

The quantity $H_{\text {sat }}$ determines the saturation field for a triangular lattice of Ising magnetic moments. If the magnetic field $H<H_{\text {sat }}$, the value of $W_{1}$ is negative and the flipping of a dot becomes favorable.

In principle, the common calculations can be performed for AFM state, as well as for any state with the superlattice of flipped dots of the same symmetry as for underlying dot lattice. This approach well describes the instability point for the AFM state for a square lattice of magnetic dots [22]. Its application for the triangular dot lattice shows that the reversal of the magnetic moment of one dot in the AFM state becomes favorable at $H \geqslant H_{\mathrm{AFM}}$, with the value $h_{\mathrm{AFM}}=1.8377$, is much higher than the instability field $h \simeq 0.7$ found numerically in the previous section. Thus, the reversal of a single magnetic moment cannot describe the instability of the AFM state observed for a triangular lattice at $h \leqslant 1$.

\subsection{Instability of AFM state through the creation of topological lines}

As we found by Monte-Carlo analysis, the AFM state looses its stability as the field increases due to the creation of topological defect lines (domain walls). This defect line corresponds to an additional zigzag line of the particles with magnetic moments, which are oriented as their neighbors, and should be normal to one of the elementary translation vectors, see figure 4 
(a)

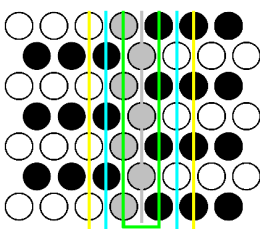

(b)

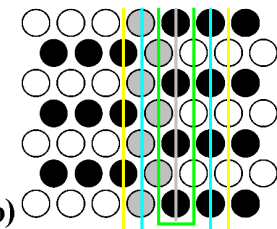

(c)

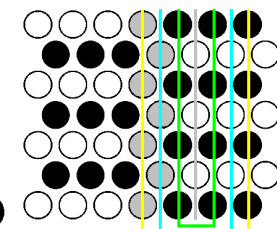

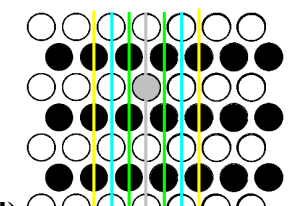

Figure 4. (Color online). (a)-(c) The structure of topological defect with the different sets of characteristic lines used for calculation of the defect energy, see details in the text. (d) The ideal AFM structure and the characteristic lines used for energy calculation. As usually, the open and closed circles denote the particles with the upward and downward magnetic moments, respectively, but the dots with upward magnetic moments within the defect line are mentioned by grey.

The importance of defect lines in thermodynamics is a well-known property of two-dimensional systems with a discrete symmetry breaking. Due to the creation of a finite density of such lines, the long range order is destroyed at finite temperature determined by the energy of the defect, see, for example, 11]. However, for frustrated AFM states, the behavior can be very unusual. In particular, there is no magnetic order for AFM Ising system with the nearest-neighbor interaction at any finite temperature $T>0$ [47, 50-52]. In general, this behavior can be explained using the defect line picture of the phase transition, with the vanishing of energy (more exactly, the free energy) of a certain linear topological defect [53].

For our system of mesoscopic magnetic particles with a dipole interaction, the effect of a magnetic field, instead of thermal effects, should be significant. The topological defect line with non-zero magnetization and having zero energy in the nearest-neighbor approximation was recently found [57]. This defect coincides with that observed in our numerical simulations, compare figure 4 and figures $3(\mathrm{a})+3(\mathrm{~d})$ above. A physical consequence of a nonzero magnetic moment is that for such a defect, an additional energy gain $m_{0} H$ per defect particle appears in the magnetic field $H$. Then, the defect energy decreases as the field increases and becomes zero at $H=H_{\mathrm{DW}} \equiv E_{\mathrm{DW}} / m_{0}$. For $H \geqslant H_{\mathrm{DW}}$, the finite density of such defects will be present in the ground state. This is exactly the scenario observed in our numerical simulations at magnetic field at the range $0.7 \div 1.5$.

In order to find the critical value of the field $H_{\mathrm{DW}}$, let us calculate the energy of the domain wall $E_{\mathrm{DW}}$. It is convenient to divide the full lattice into lines of dots parallel to the defect line, as it is shown in figure 4. The energy of the system with domain wall (per one dot in the defect line) can be presented as a sum over these lines as follows:

$$
E=E_{\mathrm{GS}}+E_{\mathrm{DW}}=-\frac{1}{2} \sum_{n} m_{0} \sigma_{n} H_{n}
$$

where $E_{\mathrm{GS}}$ is the energy of the ground state, the integer $n$ describes the distance $a_{n}$ of the given line from the defect line, $a_{n}=a n / 2, \sigma_{n}= \pm 1$ gives the sign of the moment for the $n$-th line, and $H_{n}$ is the magnetic field created on the dot in the $n$-th line by other dots in the system. To find the field $H_{n}$, it is convenient to group all other dots to pairs of lines equidistant from the $n$-th line, as it is shown for $n=0,1,2$ in figure 4(a), (b) and (c), respectively. Let us enumerate these pairs by an integer $k$ so that the distance between the $n$-th line and one component of the $k$-th pair is equal to $a k / 2$, the pairs with $k=1,2,3$ are presented in figure 4 Then, the energy of the magnetic state with a domain wall can be presented by a double sum, over $n>0$ and $k>0$.

It is easy to see that for any finite $n$, the only pairs with limited $k<n$ contribute to the energy of the state with the domain wall. For example, for the lines directly entering the defect line $[n=0$, see figure 4(a)], the contributions of two lines composing any pair cancel each other. For this line, the nonzero contribution to the energy is given by the dots from the same line, and we denote this contribution as $\varepsilon_{0}$. Then, for the line with $n=1$, only one pair gives non-zero contribution, see figure $4(\mathrm{~b})$, and the energy can be written as $\varepsilon_{0}-2 \varepsilon_{1}$. Similarly, for $n=2$, the energy is $\varepsilon_{0}-2 \varepsilon_{1}+2 \varepsilon_{2}$, see figure 4(c), and so on. Finally, the energy of the state with a domain wall is presented through $\varepsilon_{0}$ and the particular finite 
sums of the positive quantities $\varepsilon_{n}$,

$$
\varepsilon_{2 n+1}=\sum_{k=1}^{\infty} \frac{4}{\left[(n+1 / 2)^{2}+3(k-1 / 2)^{2}\right]^{3 / 2}}, \quad \varepsilon_{2 n}=\sum_{k=1}^{\infty} \frac{4}{\left(n^{2}+3 k^{2}\right)^{3 / 2}}+\frac{2}{n^{3}} .
$$

The energy of the domain wall equals the difference of the energy of the state with the domain wall, equation (4.2) and the ground state energy $E_{\mathrm{GS}}$. To find the ground state energy, it is convenient to use the same presentation by a parallel lines, see figure 4(d), and to present it by the same sums $\varepsilon_{n}$. It is clear that the energy per one dot in any line in the ground state is proportional to an infinite sum of the form $\varepsilon_{\mathrm{GS}}=\varepsilon_{0}+2 \sum_{n=1}^{\infty}(-1)^{n} \varepsilon_{n}$. Then, the domain wall energy can be found by term-by-term summation of the corresponding contributions of the form $\left[\left(\varepsilon_{0}-\varepsilon_{\mathrm{GS}}\right)+\left(\varepsilon_{0}-2 \varepsilon_{1}-\varepsilon_{\mathrm{GS}}\right)+\ldots\right] \equiv h_{\mathrm{DW}}=2 \varepsilon_{1}-4 \varepsilon_{2}+6 \varepsilon_{3}+\ldots$. The corresponding infinite series $h_{\mathrm{DW}}=-2 \sum_{n=1}^{\infty}(-1)^{n} n \varepsilon_{n}$ are sign-alternating and converge quite well. Finally, the domain wall energy per one dot $E_{\mathrm{DW}}$ can be presented as follows:

$$
E_{\mathrm{DW}}=m_{0} H_{\mathrm{DW}}, \quad H_{\mathrm{DW}}=h_{\mathrm{DW}} \frac{m_{0}}{a^{3}}, \quad h_{\mathrm{DW}}=0.70858944 .
$$

Here, we also present the characteristic value of the magnetic field, and $H_{\mathrm{DW}}=E_{\mathrm{DW}} / m_{0}$ determining the border of stability of the simple AFM state; for $H>H_{\mathrm{DW}}$, AFM state becomes unstable against the creation of domain walls. Note, that the calculated value (4.3) is in good agreement with that found by numerical simulations, but it is much lower than the field of point defect instability for AFM state, $H_{\mathrm{AFM}}=$ $1.8377 m_{0} / a^{3}$.

\subsection{Plateau description}

Monte-Carlo simulations show some peculiarities (see figure 2) in the dependence of the magnetization on the applied magnetic field in the form of plateaus, where the value of the function does not change over a wide range of the argument. These peculiarities have a simple explanation. The magnetization of the array increases at a small external field due to the formation of parallel topological defects in the form of domain walls. At some critical concentration of such walls, the resulting state is nothing but the superlattice of flipped dots which has a triangular structure that coincides with the array symmetry; see figure 3 f), (h), (i), (j). Such a superlattice transforms into a self-similar structure but with another step (lattice constant) as the applied field increases. Since the superlattice constant has discrete values $a_{\mathrm{sl}} / a=\sqrt{3}, 2, \sqrt{7}, 3,2 \sqrt{3}, \sqrt{13}, 4, \sqrt{19}, \sqrt{21}, 5 \ldots$, such a superstructure has good stability against the alteration of the external field, and magnetization can be changed only stepwise. One can see that such a structure consists of two inversely magnetized states with the lattice constants $a$ and $a_{\mathrm{sl}}$ and with the magnetization $\langle m\rangle=m_{0}-2 m_{0}\left(a / a_{\mathrm{sl}}\right)^{2}$. The value of the field of the stability loss of such a superstructure relative to the transition to another lattice constant can be easily calculated on the same principle as the field of the saturated state stability which was done above,

$$
H_{\mathrm{sl}}=h_{\mathrm{sat}} \frac{m_{0}}{a^{3}}\left[1-2\left(\frac{a}{a_{\mathrm{sl}}}\right)^{3}\right],
$$

where the multiplier 2 in the numerator responds to the change of magnetization of the dot in comparison with the saturated state and $h_{\text {sat }}=11.034176$ is the field of the transition to the saturated state. Though this effect occurs in a narrow range of the field, it leads to instability of the superlattice at a little bit smaller value of the field than it is predicted in equation (4.4).

\section{Conclusions}

The triangular regular array of magnetic particles demonstrates quite non-trivial scenario of phase transitions in the presence of an external magnetic field. The combination of two kinds of the origin of frustration, the first, present for non-bipartite triangular lattice with any kind antiferromagnetic interaction, and the second, connected with the long-range character of magnetic dipole interaction, leads to the creation of a set of linear topological defects with the growth of the magnetic field. Self-organization of 
such defects determines the magnetization process for a system within a wide range of external magnetic fields. It is worth noting an essential difference from the standard topological transitions known for an atomic spin system of low-dimensional magnets. For a system of microparticles (atomic spins) of standard low-dimensional magnets, the topological defects (vortices, domain walls, etc.) appear as a consequence of thermal fluctuations, the resulting structure being quite irregular. By contrast, in our case of the system of mesoscopic elements, the characteristic energy of the interaction of two particles is much higher than the Curie temperature. The creation/annihilation of the defects takes place at the critical value of the magnetic field, where the energy of the defect vanishes, whereas other characteristics (such as the typical interaction energy of the defects) are still finite and high, up to the values of $10^{5}$ Kelvin [22]. In our case, a quite regular pattern of these defects is realized in the system even at finite temperatures below the Curie temperature.

\section{Acknowledgements}

This work was partly supported by the State Foundation of Fundamental Research of Ukraine via grant No. F53.2/045

\section{References}

1. Weisbuch G., Complex Systems Dynamics, Addison-Wesley Publishing Company, Reading, 1991.

2. Self-organization of Complex Structures: from Individual to Collective Dynamics, Schweitzer F. (Ed.), Gordon and Breach Sci. Publ., Amsterdam, 1997.

3. Olemskoi A.I., Theory of Structure Transformations in Non-equilibrium Condensed Matter, Nova Science, New York, 1999.

4. Kosevich A.M., Ivanov B.A., Kovalev A.S., Nonlinear Waves of Magnetization. Dynamical and Topological Solitons, Naukova Dumka, Kiev, 1983 (in Russian).

5. Berezinskii V.L., Sov. Phys. JETP, 1972, 34, 610.

6. Kosterlitz J.M., Thouless J.M., J. Phys. C: Solid State Phys., 1973, 6, 1181; doi 10.1088/0022-3719/6/7/010

7. Kosterlitz J.M., J. Phys. C: Solid State Phys., 1974, 7, 1046; doi 10.1088/0022-3719/7/6/005

8. Olemskoi A.I., Sklyar I. A., Sov. Phys. Usp., 1992, 35, 455; doi 10.1070/PU1992v035n06ABEH002241

9. Olemskoi A.I., Phys. Usp., 1998, 41, 269; doi 10.1070/PU1998v041n03ABEH000377.

10. Dziarmaga J., Adv. Phys., 2010, 59, 1063; doi $10.1080 / 00018732.2010 .514702$

11. Korshunov S.E., Phys. Usp., 2006, 49, No. 3, 225; doi 10.1070/PU2006v049n03ABEH005838

12. Olemskoi A.I., Phys. Usp., 2001, 44, 479; doi 10.1070/PU2001v044n05ABEH000921.

13. Akhiezer A.I., Baryakhtar V.G., Peletminskii S.V., Spin Waves, North-Holland, Amsterdam 1968 [Nauka, Moscow, 1967 (in Russian)].

14. Hubert A., Schafer R., Magnetic domains. The analysis of Magnetic Microstructures, Springer, Berlin, 1998.

15. Baryakhtar V.G., Vitebskii I.M., Yablonskii D.A., Fiz. Tverd. Tela, 1977, 19, 347.

16. Baryakhtar V.G., Ivanov B.A., Zh. Eksp. Teor. Fiz., 1977, 72, No. 4, 1504 (in Russian) [Sov. Phys. JETP, 1977, 45, No. 4, 789]

17. Luttinger J.M., Tisza L., Phys. Rev., 1946, 70, 954; doi 10.1103/PhysRev.70.954

18. Belobrov P.I., Gekht R.C., Ignatchenko V.A., Zh. Eksp. Teor. Fiz., 1983, 84, 1097 (in Russian) [Sov. Phys. JETP, 1983, 57, 636].

19. Brankov J.G., Danchev D.M., Physica A, 1987, 144, 128; doi 10.1016/0378-4371(87)90148-8

20. Prakash S., Henley C.L., Phys. Rev. B, 1990, 42, 6574; doi 10.1103/PhysRevB.42.6574

21. Rozenbaum V.M., Ogenko V.M., Chuiko A.A., Sov. Phys. Usp., 1991, 34, 883; doi 10.1070/PU1991v034n10ABEH002525

22. Bishop J.E.L., Galkin A.Yu., Ivanov B.A., Phys. Rev. B, 2002, 65, 174403; doi 10.1103/PhysRevB.65.174403

23. Galkin A.Yu., Ivanov B.A., Merkulov A.Y., J. Exp. Theor. Phys., 2005, 101, 1106; doi 10.1134/1.2163926

24. Galkin A.Yu., Ivanov B.A., JETP Lett., 2006, 83, 383; doi $10.1134 / S 0021364006090049$

25. Skomski R., J. Phys.: Condens. Matter, 2003, 15, R841; doi 10.1088/0953-8984/15/20/202

26. Advanced Magnetic Nanostructures, Sellmyer D.J., Skomski R. (Eds.), Springer, New York, 2006.

27. Ivanov B.A., Low Temp. Phys., 2005, 31, 635; doi 10.1063/1.2008127

28. Politi P., Pini M.G., Phys. Rev. B, 2002, 66, 214414; doi 10.1103/PhysRevB.66.214414

29. Maleev S.V., Sov. Phys. JETP, 1976, 43, 1240.

30. Bruno P., Phys. Rev. B, 1991, 43, 6015; doi 10.1103/PhysRevB.43.6015

31. Ivanov B.A., Tartakovskaya E.V., Phys. Rev. Lett., 1996, 77, 386; doi 10.1103/PhysRevLett.77.386 
32. Chou S.Y., Wei M.S., Krauss P.R., Fischer P.B., J. Appl. Phys., 1994, 76, 6673; doi 10.1063/1.358164

33. Meier G., Kleiber M., Grundler D., Heitmann D., Wiesendanger R., Appl. Phys. Lett., 1998, 72, 2168; doi $10.1063 / 1.121310$

34. Au Y., Dvornik M., Davison T., Ahmad E., Keatley P.S., VansteenkisteA., Van Waeyenberge B., Kruglyak V.V., Phys. Rev. Lett., 2013, 110, 097201; doi 10.1103/PhysRevLett.110.097201

35. Ross C.A., Hwang M., Shima M., Cheng J.Y., Farhoud M., Savas T.A., Smith H.I., Schwarzacher W., Ross F.M., Redjdal M., Humphrey F.B., Phys. Rev. B, 2002, 65, 144417; doi 10.1103/PhysRevB.65.144417

36. Satoh T., Terui Y., Moriya R., Ivanov B.A., Ando K., Saitoh E., Shimura T., Kuroda K., Nature Photonics, 2012, 6, No. 10, 662; doi 10.1038/NPHOTON.2012.218

37. Kruglyak V.V., Demokritov S.O., Grundler D., J. Phys. D: Appl. Phys., 2010, 43, 264001; doi 10.1088/0022-3727/43/26/264001

38. Galkin A.Yu., Ivanov B.A., Zaspel C.E., Phys. Rev. B, 2006, 74, 144419; doi 10.1103/PhysRevB.74.144419.

39. Awad A.A., Aranda G.R., Dieleman D., Guslienko K.Y., Kakazei G.N., Ivanov B.A., Aliev F.G., Appl. Phys. Lett., 2010, 97, 132501; doi $10.1063 / 1.3495774$

40. Bondarenko P.V., Galkin A.Yu., Ivanov B.A., Zaspel C.E., Phys. Rev. B, 2010, 81, 224415; doi 10.1103/PhysRevB.81.224415

41. Sukhostavets O.V, Gonzalez J., Guslienko K.Y., Phys. Rev. B, 2013, 87, 094402; doi 10.1103/PhysRevB.87.094402

42. Verba R., Tiberkevich V., Bankowski E., Meitzler T., Melkov G, Slavin A, Appl. Phys. Lett., 2013, 103, 082407; doi $10.1063 / 1.4819435$

43. Udalov O.G., Sapozhnikov M.V., Karashtin E.A., Gribkov B.A., Gusev S.A., Skorohodov E.V., Rogov V.V., Klimov A.Yu., Fraerman A.A., Phys. Rev. B, 2012, 86, 094416; doi 10.1103/PhysRevB.86.094416.

44. Verba R., Tiberkevich V., Bankowski E., Meitzler T., Melkov G., Slavin A., IEEE Magn. Lett., 2013, 4, 4000404; doi 10.1109/LMAG.2013.2277995

45. Krawczyk M., Grundler D., J. Phys.: Condens. Matter, 2014, 26, 123202. doi 10.1088/0953-8984/26/12/123202.

46. Sellmyer D.J., Zheng M., Skomski R., J. Phys.: Condens. Matter, 2001, 13, R433; doi 10.1088/0953-8984/13/25/201

47. Gekht R.S., Sov. Phys. Usp., 1989, 32, 871; doi 10.1070/PU1989v032n10ABEH002765

48. Politi P., Pini M.G., Stamps R.L., Phys. Rev. B, 2006, 73, 020405(R); doi 10.1103/PhysRevB.73.020405.

49. Dzian S.A., Galkin A. Yu., Ivanov B.A., Kireev V.E., Muravyov V.M., Phys. Rev. B, 2013, 87, 184404; doi 10.1103/PhysRevB.87.184404

50. Wannier G.H., Phys. Rev., 1950, 79, 357; doi 10.1103/PhysRev.79.357.

51. Houtappel R.M.F., Physica, 1950 16, 425; doi 10.1016/0031-8914(50)90130-3

52. Wannier G.H., Phys. Rev. B, 1973, 7, 5017; doi 10.1103/PhysRevB.7.5017

53. Korshunov S.E., Phys. Rev. B, 2005, 71, 174501; doi 10.1103/PhysRevB.72.144417

54. Ivanov B.A., Wysin G.M., Phys. Rev. B, 2002, 65, 134434; doi 10.1103/PhysRevB.65.134434

55. Newman M.E.J., Barkema G.T., Monte Carlo Methods in Statistical Physics, Oxford University Press, Oxford, 1999.

56. Slotte P.A., Hemmer P.C., J. Phys. C: Solid State Phys., 1984, 17, 4645; doi 10.1088/0022-3719/17/26/014

57. Ivanov B.A., Kireev V.E., JETP Lett., 2009, 90, 750; doi 10.1134/S0021364009240035

\title{
Самоорганізація топологічних дефектів в трикутній гратці магнітних точок під впливом перпендикулярного магнітного поля
}

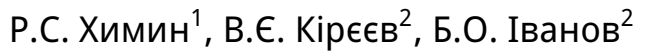 \\ ${ }^{1}$ Інститут магнетизму НАН України та МОН України, бульв. Вернадського, 36-6, 03142 Київ, Україна \\ 2 Університет Окленд, Рочестер Хіллс, 48309 Мічиган, США
}

\begin{abstract}
Періодичний масив магнітних частинок (магнітних точок), сформований в трикутну двовимірну гратку, утворює складну струтуру магнітного впорядкування. Магнітна симетрія основного стану для такої системи виявляється нижчою ніж симетрія гратки. Далекодіюча диполь-дипольна взаємодія призводить до специфічного антиферомагнітного впорядкування при малих полях, в той час як при збільшенні магнітного поля виникає серія лінійних топологічних дефектів. Самоорганізація таких дефектів визначає процес намагнічування системи в широкому діапазоні зовнішнього магнітного поля.
\end{abstract}

Ключові слова: магнітна точка, топологічний дефект 